\title{
A NEW APPROACH FOR DISPERSION CONTROL IN NANOFLUIDIC CHANNELS
}

\section{R. Qiao and N. R. Aluru}

\author{
Beckman Institute for Advanced Science and Technology \\ University of Illinois at Urbana-Champaign, Urbana, IL 61801
}

\begin{abstract}
Turns and bends, such as an L-turn or a U-turn, in micro and nanofluidic channels can induce significant dispersion leading to a low efficiency electrophoretic separation process. It has been shown that turn-induced dispersion can be lowered by optimizing the geometry of the turns. In this paper, we propose a new approach for controlling turn-induced dispersion in nanofluidic channels. The approach is based on the idea of locally controlling the $\zeta$-potential at turns and bends. An optimization algorithm was developed to search for the optimal configuration of the $\zeta$-potential near turns. Results for an L-turn and a U-turn show that the dispersion can be lowered significantly by a $\zeta$-potential variation near the turn.
\end{abstract}

\section{INTRODUCTION}

There has been growing interest in developing miniaturized fluidic devices for transport, separation and detection of biosamples. In many of these devices, capillary electrophoresis is used for sample separation, and electroosmosis is used for sample transport. In several cases, both these processes occur together. Prior studies have shown that a longer channel can improve the separation efficiency. In applications like lab-on-a-chip where the device size is very small, turns must be introduced to integrate a long channel within the small area of a single chip. However, it has been found that such turns could induce serious dispersion, which may negate the effect of using a long channel.

Griffiths et. al. [1] and Molho et. al. [2] suggested the development of optimized turns to minimize the dispersion. In both these works, numerical methods were used to find the optimal geometrical configuration of the turns. Experimental results show that such an approach is feasible [2]. However, fabrication of the optimized geometry may not always be possible because of manufacturing as well as other considerations. In this work, we propose to control the turn-induced dispersion by introducing a localized $\zeta$-potential variation. In this method, the $\zeta$-potential on channel walls near the turn is controlled and the turn geometries are not altered. This approach provides an alternative when optimized turn geometry is difficult to implement in practice. Though flow control using localized $\zeta$-potential variations has been difficult in the past, recent results suggest that it is possible to use such an approach. For example, it was reported in [4] that a localized $\zeta$ potential control (through surface patterning) can be employed to produce nonaxial flow in microchannels.

\section{MATHEMATICAL MODEL}

Fluid flow due to electrical potentials, also referred as electroosmotic transport, is described by the Laplace equation, the Poisson-Boltzmann equation and the incompressible Navier-Stokes equations. A detailed description of the electroosmotic transport model can be found in [3]. The model includes:

$$
\begin{aligned}
& \nabla^{2} \phi=0 \\
& \nabla^{2} \Psi=\frac{1}{\lambda^{2}} \frac{R T}{F} \sinh \left(\frac{F \Psi}{R T}\right) \\
& \nabla \cdot \boldsymbol{u}=0 \\
& \mu \nabla^{2} \boldsymbol{u}-\nabla P+\boldsymbol{F}=0 \\
& \boldsymbol{F}=\varepsilon \nabla \phi \nabla^{2} \Psi
\end{aligned}
$$

The Laplace equation (1) describes the potential in the electrokinetic geometry because of applied potentials and the Poisson-Boltzmann equation (2) describes the potential $(\Psi)$ in the device because of the $\zeta$-potential at the solution-capillary interface. Equations (3) and (4) describe the fluid flow in the device because of the applied and $\zeta$-potential. Specifically, equation (3) is the incompressible continuity equation and equation (4) is the Stokes equation. In equation (4), $\boldsymbol{F}$ is the body force, the definition of which is given in equation (5). The mathematical model stated in equations (1) - (5) along with the appropriate boundary conditions describes the two-dimensional electroosmotic problem.

In some previous works (e.g. [1] and [2]), a potential flow is assumed and fluid velocity in electroosmotic flow is assumed to be proportional to the gradient of the applied electric field. This has been justified under several assumptions, of which the most important one may be that the $\zeta$-potential in the entire system must be uniform. Because we intentionally introduce non-uniform $\zeta$ potentials on the wall near turns, a full set of flow and electrokinetic equations (1)-(5) need to be solved to obtain the flow field instead of using the simpler potential flow theory.

Dispersion can be caused by both advection and diffusion [1] In this paper, for simplicity, we consider only the dispersion caused by advection and neglect the diffusion-induced dispersion. The approach can, however, be extended in a straightforward manner to account for diffusion. When diffusion is taken into account, the optimization approach introduced here need not be changed, though the optimal configuration of the $\zeta$-potential on the wall might change. The dispersion is calculated by tracking particles released at the upstream of the turn. The path of these particles is obtained by integrating:

$$
\frac{\partial \mathbf{x}}{\partial t}=\mathbf{u}
$$

where $\boldsymbol{u}$ is the velocity computed by solving equations (1)-(5). Here, we assume that the velocity of the particles is the same as the local fluid velocity.

\section{NEW CONTROL STRATEGY: LOCALIZED ZETA- POTENTIAL VARIATION}

In nanodiameter channels, turns can induce serious dispersion. Shown in Figure 1 is the distribution of particles after passing an L-shaped turn. These particles were released at the upstream of the turn with the same position in the $x$-direction. It can be seen that a serious dispersion occurs as the particles go through the $90^{\circ}$ turn. 
The dispersion stems from the fact that the particles near the inner channel wall travel a shorter distance compared to the particles near the outer channel wall. The differing velocity of the particles also contributes to the dispersion. An important observation in Figure 1 is that the particles are no longer in the symmetrical position with respect to the centerline of the channel after they have gone through the L-shaped turn.

One approach to minimize such dispersion is to make the particles travel approximately the same distance as they go through the turn. An alternative approach is to alter the velocity of the particles such that particles travelling longer distances move faster and particles moving shorter distances move slower. A combination of the two approaches can also be employed to minimize the dispersion. An example of the first approach is the work described in [1] and [2], where the geometry of the turn is optimized such that the particles travel approximately the same distance at roughly the same velocity.

We propose a new method in which by varying the $\zeta$-potential at the inner wall of the turn, we can change both the migration paths of these particles as well as their velocities without changing the geometry of the turn. By varying $\zeta$-potential, we change the electric field, which in turn alters the migration path and the velocity of the particles. Thus, by controlling the $\zeta$-potential on the inner wall near the turn, the particle dispersion can be minimized.

In this research, we have developed a two-stage control strategy to minimize the particle dispersion. The control scheme is illustrated in Figure 2 for an L-turn. In the first stage, the $\zeta$ potential near the turn is controlled such that the particles are at a symmetrical position with respect to the centerline of the channel after they have passed the turn. In the second stage, the $\zeta$-potential is controlled such that the particle dispersion is minimized. For an L-shaped turn, the second control step implies that the vertical position ( $y$-coordinate) of the particles is approximately the same.

Figure 3 shows the position of the particles after passing the turn when only the first stage control is employed. It can be seen that the particle profile is almost symmetric with respect to the centerline of the channel. However, the particles near the channel wall lag behind the particles in the central portion of channel, indicating that the dispersion is still very high. Such a situation can be improved by employing the second stage control. Figure 4 shows the position of the particles after passing the turn. Note that the dispersion is much less than that observed in Figure 1. Even in Figure 4, the particles very near the wall still lag behind the particles in the central portion of channel. This can be explained by the fact that if a particle is within or near the Debye layer (the Debye length is set to be $2.5 \mathrm{~nm}$ ), it has a smaller velocity compared to a particle that is in the central portion of the channel. Even in a straight channel, where there is no bend or a turn, such dispersion, where the particles in the Debye layer lag behind the particles in the central portion of the channel, is observed. Figure 5 shows the streamlines near the turn when the two-stage control is applied. It can be seen that though the geometry of the turn is not altered, the migration path of the particles is changed.

\section{APPROACH}

The approach used to minimize the particle dispersion is discussed in this section. In particular, the objective functions developed for each of the control steps are described. For the Lshaped channel example discussed in the previous section, for the first control-step, where the objective is to make the particle profile symmetric with respect to the center line of the channel, the following objective function is employed:

$$
F_{1}=\sum_{i=1}^{n}\left|w_{i}\left(y_{i}-y_{2 n+1-i}\right)\right|
$$

In the development of equation (7), $2 n$ particles are assumed to be released from the upstream of the channel. $y_{i}$ refers to the $y$ coordinate of the $i$ th particle after the particle has gone through the turn (see Figure 2). Note that in equation (7), the first particle is assumed to be the particle closest to the lower or the bottom channel wall and the $2 n$th particle is the particle closest to the top or the upper channel wall. $w_{i}$ is the weight attached to each particle. As demonstrated in Figure 3, minimization of equation (7) leads to a symmetric particle profile as the particles go through the turn. The minimization of equation (7) can be accomplished by employing a standard optimization algorithm [5].

The objective of the second control step is to minimize the dispersion or scatter between the particles. The objective function for the second control step is taken to be:

$$
F_{2}=\sum_{i=1}^{2 n} w_{i} \frac{\left|y_{i}-\bar{y}\right|^{2}}{2 n}
$$

$w_{i}$ is again the weight assigned to each particle. $\bar{y}$ is the mean vertical position of all the particles and $y_{i}$ is the $y$-coordinate of the $i$ th particle.

Equations (7) and (8) can be minimized by treating the $\zeta$ potential at each mesh point on the channel wall as a control parameter. While this may yield the best results, it may not be a very practical situation. Hence, we use the following parameters as control variables to minimize the dispersion in an L-shaped turn:

1. For the first control step, it is assumed that the $\zeta$ potential varies linearly within the control region i.e. $\zeta$-potential varies linearly on $O A$ and $O B$ (see Figure 2). The $\zeta$-potential at points $A$ and $B$ is assumed to be $\zeta_{0}\left(\zeta_{0}\right.$ is the known uniform $\zeta$ potential on the channel walls when no control strategy is applied) and the $\zeta$-potential at point $O$ is the unknown. The length of the regions $O A$ and $O B$ is assumed to be the same and the $\zeta$-potential variation on $O A$ and $O B$ is as shown in Figure 6. The two unknowns to be determined are the $\zeta$-potential at point $O$ and the length of region $O A$ or $O B$. These unknowns are determined by minimizing equation (7). The $\zeta$-potential at point $A$ and the length of $O A$ determined by the optimization algorithm are shown in Figure 7.

2. For the second control step, we assume that the length of the region $B D$ or $B^{\prime} D^{\prime}$ is equal to the width of the channel and the $\zeta$-potential is constant on $B D$ or $B^{\prime} D^{\prime}$. The unknown in this case is the constant $\zeta$-potential on $B D$ or $B^{\prime} D^{\prime}$ and this can be computed by minimizing equation (8). We chose the length of $B D$ to be the width of the channel just to keep the approach simple. It is, of course, possible to treat the length of $B D$ also as an unknown. The optimal $\zeta$-potential on $B D$ (and $B^{\prime} D^{\prime}$ ) is determined to be $1.3 \zeta_{0}$.

The results for the L-turn suggest that the $\zeta$-potential need to be controlled only at positions very close to the turn, e.g. a few channel widths of the turn. Figure 4 suggests that these control strategies are effective in significantly lowering the dispersion.

\section{RESULTS AND DISCUSSION}

The two-stage control approach can be applied to minimize dispersion in other electrokinetic geometries e.g. a U-turn. Shown in Figure 8 is a sketch of the control strategy used for a U-turn. 
Similar to an L-turn channel, we have assumed that the $\zeta$-potential distribution on $o-a$ and $o^{\prime}-b$ is linear and the $\zeta$-potential along $o-o^{\prime}$ and $b-c$ is constant. In addition, the length of the second stage control region (line $b-c$ and $b^{\prime}-c^{\prime}$ in Figure 8 ) is set to be the channel width. Using the algorithm described above, the optimal configuration of the $\zeta$-potential can be found. Figure 9 shows the dispersion of the particles before the control strategy was applied and Figure 10 shows the dispersion of particles after the control strategy was enforced. It can be seen that the dispersion has been lowered significantly with the two-step control strategy.

One important issue behind the control strategies described here is whether one can manipulate the $\zeta$-potential in the way that the optimization algorithm has suggested? Changing the $\zeta$-potential on the channel walls has been difficult in the past, but recently there has been a lot of work done that makes it possible to change the $\zeta$-potential at walls in a desired way. Various techniques such as altering buffer $\mathrm{pH}$, adding buffer additives, coating of channel wall and applying external voltages across the channel wall have been proposed. Of these approaches, coating of channel wall and applying external voltages can be used to change the $\zeta$-potential on the walls locally. Flow control by coating the channel wall has been reported recently [4]. Important progress has also been made in controlling the $\zeta$-potential by applying external voltages. The $\zeta$ potential can now be changed by applying low external potentials (a few hundred volts), whereas in the past this required very high external voltages [6].

\section{CONCLUSIONS}

In this paper a novel technique is introduced to control the dispersion induced by turns in nano-channel systems. In the proposed technique, $\zeta$-potential on the wall near the turn is controlled to minimize the dispersion, and the geometry of the turn is not altered. An optimization algorithm has been developed to search for the optimal $\zeta$-potential distribution. Our results on dispersion control in an L-turn and U-turn suggest that:

1. The dispersion caused by the presence of a turn can be lowered significantly by locally altering the $\zeta$-potential distribution at the turn.

2. To minimize the dispersion, $\zeta$-potential need to be altered only within a few channel widths near the turn.

Though the discussion is focused on nano-channel systems, the technique can be applied to dispersion control in micro-channel systems. In addition, the motion of particles due to diffusion, which is neglected in this paper, can be accounted for in a straightforward manner. When diffusion is taken into account, typically, a different $\zeta$-potential distribution is necessary to minimize the particle dispersion.

\section{ACKNOWLEDGMENT}

This work was supported by a grant from DARPA under agreement number F30602-98-2-0718. Travel support has been generously provided by the Transducer Research Foundation and by the DARPA MEMS and DARPA BioFlips programs.

\section{REFERENCES}

1. S.K. Griffiths and R.H. Nilson. "Low-dispersion turns and junctions for microchannel systems", Anal. Chem., 73, 2 (2001).
2. J. Molho, A.Herr and et al, "Optimization of turn geometries for microchip electrophoresis", Anal Chem., 73, 6, (2001).

3. M. Mitchell, R.Qiao and N.Aluru. "Meshless analysis of steadystate electro-osmotic transport", Journal of Microelectomechanical Systems, 9 (2000).

4. A.D. Stroock, M.Wech and et al, "Patterning electro-osmotic flow with patterned surface charge", Phys Rev Lets, 84, 15 (2000).

5. M.T. Heath. "Scientific computing: an introductory survey", WCB/McGraw-Hill, 1997.

6. N.A. Polson and M.A. Hayes. "Electroosmotic flow control of fluids on capillary electrophoresis microdevice using an applied external voltage", Anal. Chem., 72, 5 (2000).

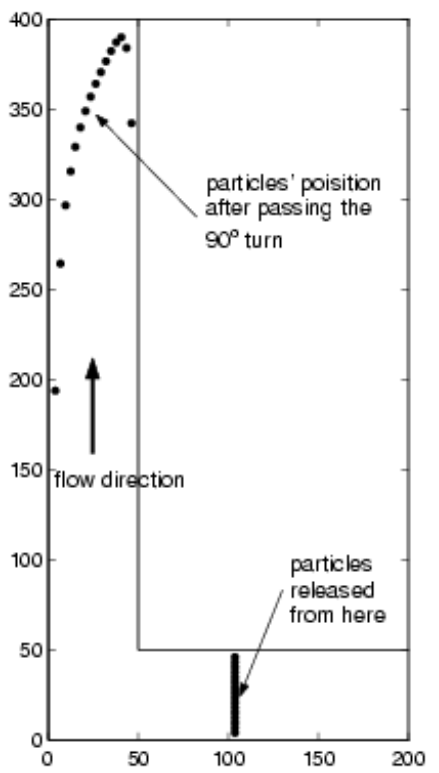

Figure 1. Distribution of particles after passing the $90^{\circ}$ turn.

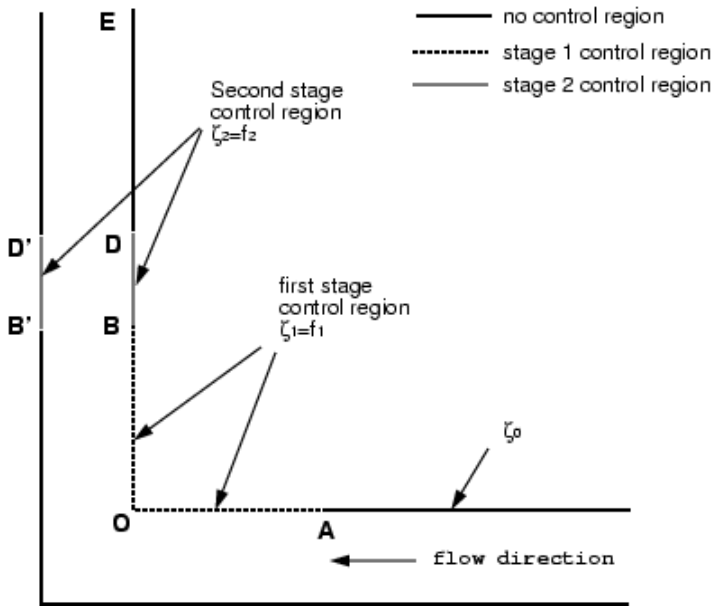

Figure 2. Two-stage control strategy to minimize the particle dispersion in a $90^{\circ}$ turn. 


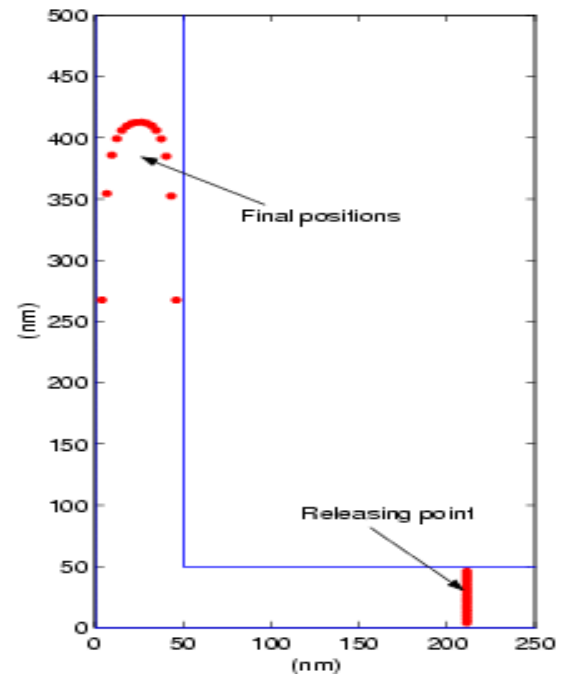

Figure 3. Distribution of particles after passing through the $90^{\circ}$ turn. Only first stage control is enforced. Observe that the particle positions are fairly symmetric about the centerline of the channel.

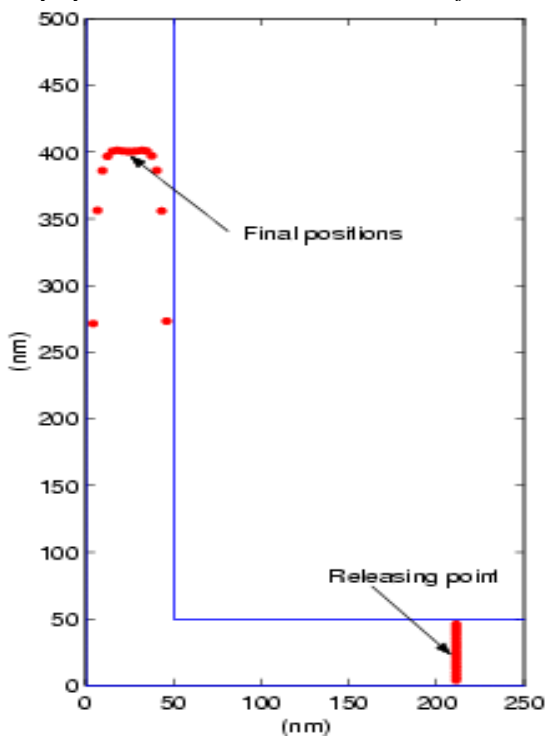

Figure 4. Distribution of particles after passing through the $90^{\circ}$ turn. Both first and second stage control are enforced. Observe that the particle dispersion is significantly lowered.

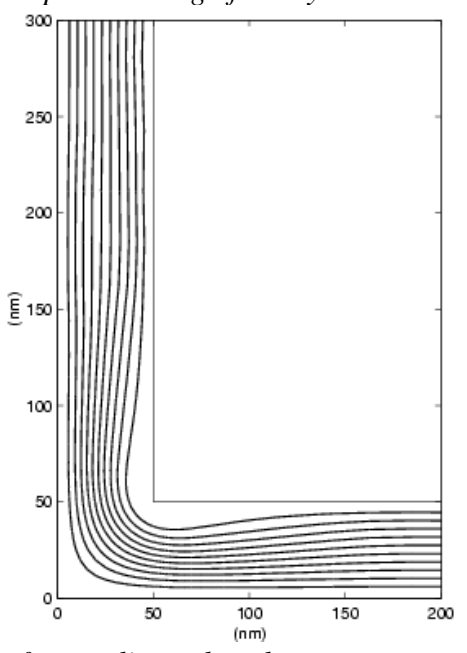

Figure 5. Plot of streamlines when the two-stage control approach is employed for an L-turn.

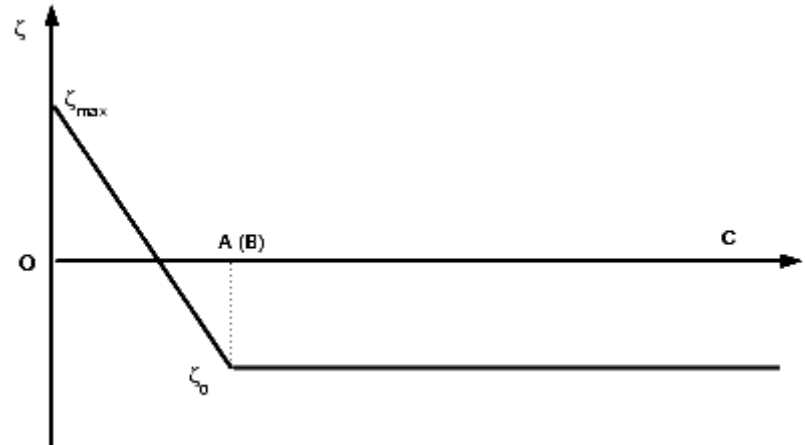

Figure 6. Linear ל-potential distribution is assumed on $O A$ and $O B$. Positions of points $O, A, B$ and $C$ are as shown in Figure 2.

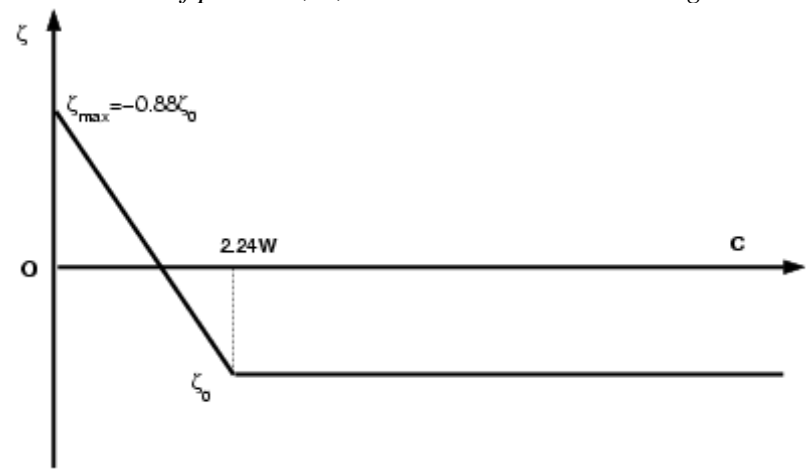

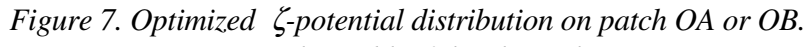
$W$ is the width of the channel.

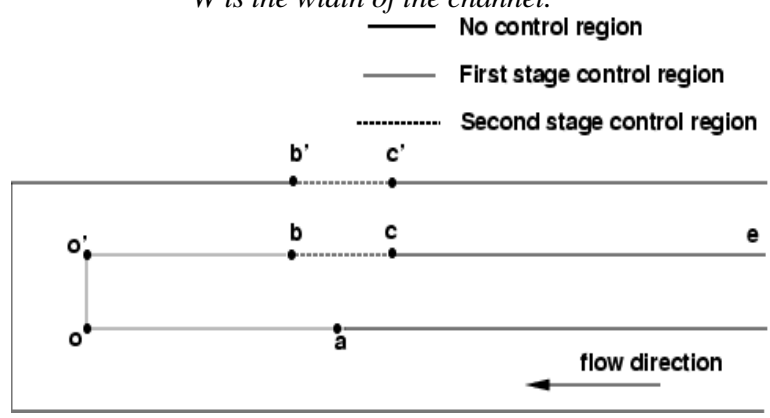

Figure 8. Sketch of a two-stage control strategy to minimize dispersion in a $U$-turn.

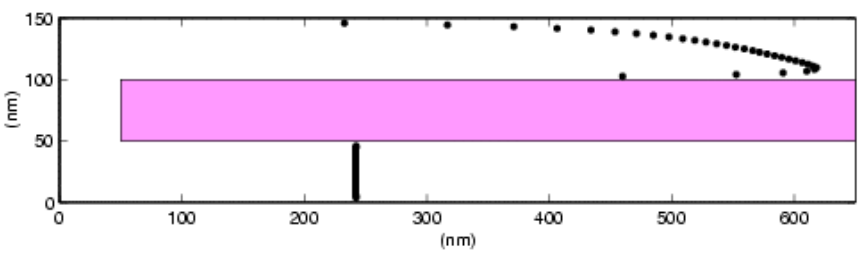

Figure 9. Distribution of particles after passing through a U-turn. No control algorithm was applied.

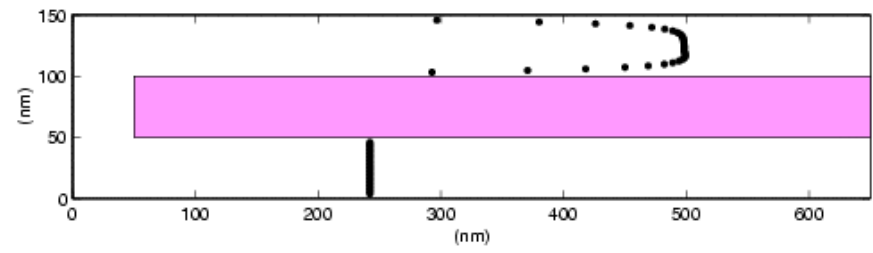

Figure 10. Distribution of particles after passing through a U-turn. A two-step control strategy as described in Figure 8 is applied. 